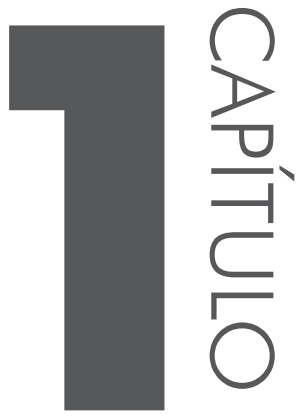

\title{
CONTROLE SEM FIO POR DISPOSITIVOS MÓVEIS DE PRÓTESE BIÔNICA DE MÃO
}

Lísias Camargo ${ }^{1}$ Marcelo Stoppa ${ }^{1}$

Agradecimentos: Os autores agradecem o suporte financeiro do CNPq por meio do edital MCTI-SECIS-CNPq-84-2013, proc. 458649/2013-9.

Resumo: Este artigo apresenta etapas da construção de um aplicativo para os Sistemas Operacionais Android e, posteriormente, iOS, com o intuito de enviar sinais de controle por meio de comunicação sem fio a uma prótese de mão de baixo custo produzida em impressora 3D. O tráfego de dados de controle ocorre via Bluetooth entre dispositivo móvel e o hardware de controle da prótese. A prótese utilizada é dotada de cinco dedos, cada um deles comandados por servomotores,

1 Unidade Acadêmica Especial de Matemática e Tecnologia, Universidade Federal de Goiás - Regional Catalão, Catalão, Brasil. 
em movimento de flexão e extensão, num total de 17 graus de liberdade. Por meio do dispositivo móvel, o usuário envia dados ao controlador, composto por uma placa Arduino e um módulo Bluetooth, que os interpreta e aciona os servomotores, produzindo o movimento dos dedos na prótese.

Palavras-chave: Tecnologia assistiva. Aplicativos. Sistemas embarcados móveis. Prótese de mão.

Abstract: This article presents the application construction stages for Android and later iOS operating systems, those send signals of control via wireless communication to a low-cost hand prosthesis produced by 3D printer. The control data traffic takes place via Bluetooth between the mobile device and prosthesis control hardware. The used prosthesis is equipped with five fingers, each of which, controlled by servomotors in flexion and extension movements, a total of 17 degrees of freedom. Through the mobile device, the user sends data to the controller, composed of an Arduino Shield and Bluetooth card who plays this and triggers the servomotors, producing the movement of the fingers on the prosthesis.

Keywords: Assistive Technology. Applications. Mobile embedded systems. Hand prosthesis.

\section{INTRODUC̣ÃO}

As redes sem fio mudaram a maneira como se manipula os dados virtuais. Observa-se que o seu surgimento não é novo: essa comunicação, como aponta Tanenbaum (2003), surgiu na Inglaterra em 1901 com contribuição do físico Italiano Guglielmo Marconi, que demonstrou como funcionava um telégrafo sem fio através da transmissão de informações de um navio até o litoral no Canadá.

Segundo Kurose e Ross (2010), posterior a esse período, em 1969 iniciou-se um projeto de Ciência de Computadores no Advanced Research Projects Agency Agência de Projetos de Pesquisa Avançada (ARPA), liderado por J. C. R. Licklider e Lawrence Roberts, que foi considerado a primeira rede de computadores. Esse projeto conectava quatro nós em rede, e sua primeira utilização foi através de um login remoto entre o sistema da Universidade da Califórnia em Los Angeles e o Instituto de Pesquisas de Stanford, o que mais tarde permitiu o surgimento da internet.

As contribuições de Marconi, Licklider e Roberts levaram à evolução da comunicação sem fio, possibilitando hoje que uma pessoa consiga se conectar à internet em uma grande variedade de lugares e aparelhos utilizados.

A comunicação sem fio trouxe avanços em diversas áreas do conhecimento. Dentre elas pode-se citar a Tecnologia Assistiva (TA). O termo Assistive Technology, traduzido no Brasil como Tecnologia Assistiva, foi criado em 1988 como importante elemento jurídico dentro da legislação norte-americana conhecida 
como Public Law 100-407 e foi renovado em 1998 como Assistive Technology Act (SARTORETTO; BERSCH, 2014).

A Tecnologia Assistiva, dentre outros aspectos, assume grande importância no contexto de desenvolvimento de próteses, facilitando a vida das pessoas que possuem algum tipo de deficiência motora. A deficiência, de modo geral, é marcada pela perda de uma das funções do ser humano, seja ela física, psicológica ou sensorial (SCHIRMER et al., 2007).

De acordo com Hunold et al. (2014), a TA é a área do conhecimento que envolve desenvolvimento de serviços e produtos com o objetivo fundamental de incluir a pessoa com deficiência na sociedade. Imerso nos estudos sobre TA, o objetivo do presente trabalho é demonstrar as etapas da criação de um aplicativo que será disponibilizado nas plataformas Android e iOS para controle de uma prótese biônica de mão.

Como dispositivo de controle, é utilizada uma placa Arduino, que, devido às suas características e funcionalidades, é peça-chave na comunicação. O hardware, por meio de comunicação sem fio, envia sinais de controle via protocolos específicos aos servomotores da prótese. São apresentados os resultados parciais, as dificuldades encontradas durante a implementação e propostas de melhorias futuras.

\section{ARDUINO}

Para a realização deste trabalho, foi utilizada uma placa Arduino Uno, que atua como mediadora entre os comandos de dispositivo móvel e a prótese biônica de mão.

O Arduino é uma pequena placa microcontrolada (ATMega) e porta de conexão Universal Serial Bus (USB) que permite a ligação com um computador. Além disso, possui diversos outros terminais que permitem a conexão com dispositivos externos, como motores, relés, sensores luminosos, LEDs e alto-falantes, dentre outros (MONK, 2013). Essa placa foi proposta em 2005 no Interaction Design Institute, na cidade de Ivrea, na Itália, pelo professor Banzi e pelo pesquisador Cuartielles para que seus alunos pudessem utilizá-la em projetos. O intuito era desenvolver um microcontrolador com custo reduzido e uma plataforma utilizada facilmente por qualquer pessoa (EVANS; NOBLE; HOCHENBAUM, 2013).

De acordo com Bastos, Borges e D’ Abreu (2010), o software do Arduino é baseado no Processing, sendo utilizada uma linguagem semelhante à linguagem de programação C, mas desenvolvida no Massachusetts Institute of Technology Instituto de Tecnologia de Massachusetts (MIT).

Para McRoberts (2011), a maior vantagem do Arduino sobre outras plataformas de desenvolvimento de microcontroladores é a facilidade de sua utiliza- 
ção, sobre a qual o autor ressalta que pessoas que não são da área técnica podem, rapidamente, aprender o básico e criar seus próprios projetos em um intervalo de tempo relativamente curto. Além disso, o software de programação é multiplataforma, o que significa que pode ser executado em Windows, Linux ou iOS.

Outro fato interessante do Arduino é que ele é um projeto gratuito. Margolis (2011) enfatiza que o software é open source, o que facilita a sua disseminação, uma vez que qualquer pessoa pode alterar sua estrutura e até mesmo sua programação. Uma grande vantagem está no custo de aquisição da placa, que é relativamente baixo, além do fato de existir uma comunidade ativa online de apoio acessível em todo o mundo através dos fóruns de discussão.

Assim, desenvolvedores e técnicos podem solucionar problemas de forma mais rápida e criar soluções acompanhando a realidade de projetos atuais. A Figura 1 exibe a placa Arduino Uno utilizada no controle da prótese biônica de mão.

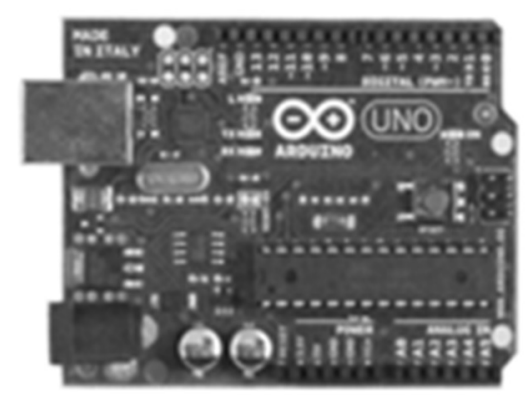

Figura 1. Arduino Uno. Fonte: Margolis (2011).

Por outro lado, o Arduino apresenta algumas limitações. Como exemplo, em relação à programação, Lima e Villaça (2012) criticam que um programa escrito em seu Ambiente de Desenvolvimento - Integrated Development Environment (IDE) - consome mais memória que um programa escrito puramente em linguagem $C$ através de outro ambiente.

Outro fator diz respeito à facilidade para pirataria de hardware com a produção de placas paralelas no mercado, uma vez que é um projeto open source, como explica Simões (2015). Isso significa que a placa pode ser adaptada, porém, é necessário utilizar a licença General Public License (GPL) de empresas licenciadas.

Banzi (2013) ressalta que, para apoiar o projeto Arduino, os clientes devem estar cientes de que placas paralelas não contribuem em termos financeiros ou em documentação de software, e ressalta ainda que os fabricantes oficiais atuais são SmartProjects, na Itália, Sparkfun, nos EUA, e DogHunter, em Taiwan/China. Para ele, esses são os únicos fabricantes que têm permissão para usar o logotipo do Arduino em suas placas. 


\section{PADRÕES DE COMUNICAC̣ÃO}

Quando surgiram as redes de computadores, não havia uma padronização para o compartilhamento de dados. Torres (2001) destaca que pelo fato de no início não ser possível realizar a comunicação entre redes de fabricantes distintos, a International Standards Organization (ISO) desenvolveu um modelo para facilitar a conexão de sistemas de computadores.

A solução proposta pela ISO foi nomeada como Open Systems Interconnection (OSI), e seu intuito principal era que os fabricantes pudessem criar protocolos a partir desse modelo. O modelo OSI foi proposto em sete camadas com o objetivo de padronizar o envio e recebimento de informações, conforme ilustra a Figura 2.

\begin{tabular}{ccc}
\hline 7 & Aplicação \\
\cline { 1 - 1 } 6 & Apresentação \\
\cline { 1 - 1 } 5 & Sessão \\
\hline 4 & Transporte \\
\cline { 1 - 1 } 3 & Rede \\
\cline { 1 - 1 } 2 & Link de dados \\
\hline 1 & Física \\
\hline
\end{tabular}

Figura 2. 0 modelo OSI proposto pela ISO. Fonte: Torres (2001).

Entre as comunicações que surgiram com o desenvolvimento e a padronização das redes de computadores propostas pela ISO, destacam-se as sem fio: Bluetooth, ZigBee e infravermelho - Infra Red (IR). Santos et al. (2012) descreveram o Bluetooth como sendo um protocolo de comunicação sem fio projetado para baixo consumo de energia e baixo alcance, com o propósito de eliminar as conexões físicas entre dispositivos de diferentes fabricantes. Em relação à tecnologia ZigBee, Brito, Oliveira e Caetano (2009) apontam que esta foi desenvolvida pela ZigBee Alliance e objetiva um conceito de transmissão de dados para redes de sensores sem fio de curta distância. Já o infravermelho, de acordo com Lucas (2015), é um tipo de radiação eletromagnética, como ondas de rádio, radiação ultravioleta, raios X e micro-ondas. Esse tipo de comunicação é muito encontrado em televisores, controles remotos e equipamentos eletrônicos.

Santos et al. (2012) realizaram uma pesquisa com os tipos de comunicação citados anteriormente, onde propuseram o desenvolvimento de um protótipo de veículo teleoperado por aplicativo móvel. A partir daí, os autores puderam comparar as tecnologias sem fio Bluetooth, ZigBee e infravermelho e apontaram algumas vantagens do ZigBee em relação às outras duas, como a 
quantidade de dispositivos em rede, o alcance em metros e o monitoramento e controle de dados transmitidos. No entanto, os autores puderam concluir que a comunicação Bluetooth apresenta maior amplitude de aplicação, devido ao grande número de aparelhos no mercado que oferecem suporte a essa tecnologia, ressaltando que há uma carência de dispositivos móveis com interface para ZigBee e IR.

Além disso, o IR tem a desvantagem de perda de dados diante de obstáculos físicos. Os dados da pesquisa de Santos et al. (2012) são exibidos na Tabela 1. Brito et al. (2009) também destacaram que o Bluetooth possui taxa de transferência, capacidade de armazenamento e consumo de energia mais altos, já que visam aplicações em dispositivos pessoais com tamanhos maiores do que os utilizados pelas redes ZigBee.

Tabela 1. Comparativo entre as tecnologias sem fio ZigBee, Bluetooth e infravermelho

\begin{tabular}{cccc}
\hline & ZigBee & Bluetooth & IR \\
\hline $\begin{array}{c}\text { Dispositivos em rede } \\
\text { Transmissão de }\end{array}$ & $\sim 65.000$ & 7 & 0 \\
dados (kbps) & 250 & 1000 & 10 \\
Alcance (m) & 100 & $10-100$ & 5 \\
$\begin{array}{c}\text { Características de } \\
\text { aplicação }\end{array}$ & $\begin{array}{c}\text { Monitoramento e } \\
\text { controle }\end{array}$ & $\begin{array}{c}\text { Transmissão de } \\
\text { dados sem fio }\end{array}$ & $\begin{array}{c}\text { Transmissão de } \\
\text { dados sem fio } \\
\text { Consumo (dias) }\end{array}$ \\
\hline
\end{tabular}

Fonte: adaptada de Santos et al. (2012).

Diante do exposto, optou-se pela utilização da tecnologia Bluetooth para controle da prótese biônica de mão, que se aponta como a melhor opção até o presente momento quando se trata da comunicação sem fio entre aplicações móveis e interface física.

Pode-se descrever a arquitetura da tecnologia Bluetooth, conforme afirma Priess et al. (2003); a banda base da arquitetura é a camada física do Bluetooth, que é responsável, entre outras funções, pela criação das piconets e dos enlaces. Os autores descrevem que uma rede composta de várias piconets representa uma scatternet, em que a piconet se refere a um conjunto de até oito integrantes ativos, e estes compartilham a mesma região de frequência. Um dos dispositivos assume o papel de mestre e os outros se comportam como escravos (Figura 3). 


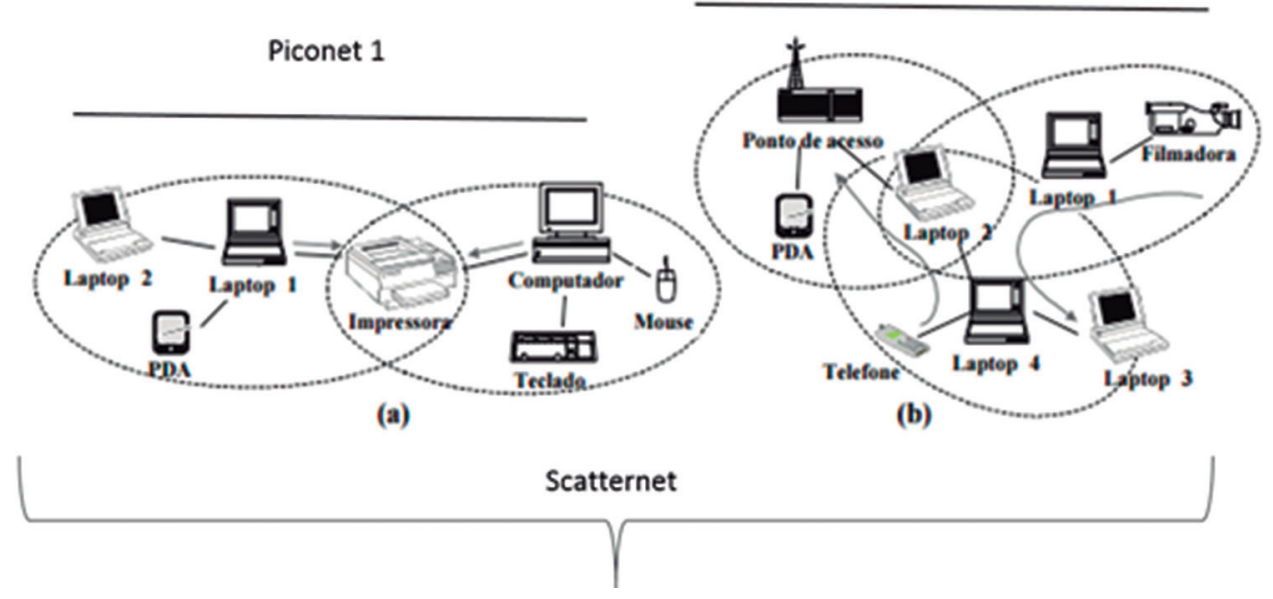

Figura 3. Exemplos de cenários de scatternets. Fonte: adaptada de Priess et al. (2003).

\section{PRÓTESES DE MÃO}

Várias pesquisas têm sido desenvolvidas com o objetivo de implementar próteses biônicas, e seu uso não é novo. Desde a antiguidade, de acordo com Hunold et al. (2014), elas vêm sendo utilizadas na tentativa de dar aos indivíduos que não têm um ou mais membros uma melhor aparência e ajudar em algumas tarefas do dia a dia. Os egípcios foram os pioneiros no uso de tecnologia de próteses, sendo que os membros protéticos eram feitos de fibras, e tinham uma função mais psicológica do que estética.

Norton (2007) afirma que a primeira prótese foi descoberta em uma múmia egípcia: uma prótese do dedo do pé, datada por volta de 300 a.C. Aponta também que, em 1858, foi descoberta uma perna artificial de bronze e ferro em Cápua, na Itália, a qual possuía um núcleo de madeira, aparentemente para um amputado abaixo do joelho.

Ainda de acordo com Norton (2007), foi no período da Renascença que as próteses evoluíram. Esse período marcou o início de novas perspectivas de arte, filosofia, ciência e medicina. As próteses eram geralmente feitas de ferro, aço, cobre e madeira, mas ainda assim tinham função mais estética do que funcional.

O que se percebe é que o uso de próteses continuará evoluindo, pois de acordo com Dellon e Matsuoka (2007), na década de 1950, apenas 4,9\% da população atingia a idade de 65 anos. Em 2007, quase 20\% tinha mais de 65 anos, e esse cenário está previsto para ser superior a $35 \%$ em 2050. Assim, a assistência por dispositivos robóticos para suprir as necessidades dessas pessoas tende a ser 
maior nos próximos anos. Com essa demanda, projetistas de robótica buscam adequar a realidade dos indivíduos com necessidades especiais.

Weir (2004) aponta que, com o tempo, as próteses passaram por mudanças, mas o desafio é criar meios que possibilitem que as mesmas estejam acessíveis e disponíveis a uma grande quantidade de pessoas. Mesmo com essa preocupação e com o surgimento dessa grande demanda, nota-se que, comercialmente, existe disponibilização de próteses funcionais, mas com alto custo.

Como exemplo, na Escócia foi desenvolvida, pela companhia Touch Bionics, uma prótese biônica de mão chamada I-Limb ${ }^{\circledR}$. Essa prótese possui, em sua estrutura, motores que permitem a mão dobrar as articulações de cada dedo, e também voltar a posição normal depois de um tempo de inatividade (Figura 4).

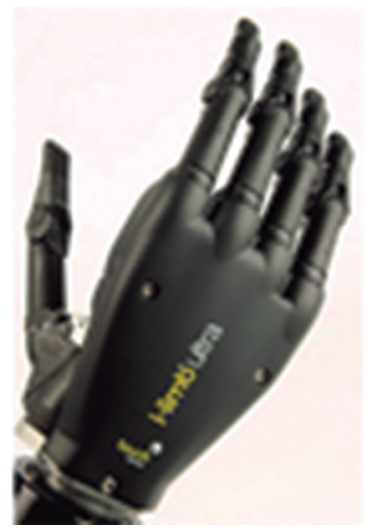

Figura 4. Prótese l-Limb. Fonte: www.touchbionics.com.

A empresa disponibiliza também um aplicativo móvel denominado Biosim ${ }^{\circledR}$ para controle de sinais via celular iPhone ${ }^{\circledR}$. Contudo, o que se observa é o alto custo para aquisição da prótese, dificultando a obtenção desta pela maioria da população. Segundo Webster (2013), da CNN, incluindo a instalação e treinamento, a prótese I-Limb ${ }^{\circledR}$ custa hoje em torno de US\$100.000.

Outra prótese disponibilizada comercialmente é a Michelangelo ${ }^{\circledR}$, que é uma prótese biônica desenvolvida pela empresa Otto Bock no Canadá. Segundo Amorim e Garrido (2010), ela é consequência de mais de dez anos de pesquisa e é controlada por meio de dois eletrodos de contato cutâneo colocados nos músculos tensor e extensor que, quando contraídos, enviam um potencial de ação em um milionésimo de segundo à placa processadora. Internamente, a mão é construída de aço e duralumínio de alta resistência que substituem a estrutura endoesquelética dos ossos e das articulações humanas. Externamente, é recoberta por macios elastômeros de silicone, assim como outros plásticos de alta tecnologia que plagiam os músculos e os tendões. 
Por outro lado, Hunold et al. (2014) descrevem que mesmo as próteses mais caras não conseguem reproduzir todos os movimentos necessários para a realização de algumas tarefas do dia a dia, como, por exemplo, abrir e fechar uma torneira. Para os autores, esses fatos limitam a autonomia e a independência da pessoa com amputação.

Assim, é necessário repensar novas formas de criação voltadas à realidade do amputado. Para Weir (2004), a questão analisada pelos projetistas de robótica é como interligar uma prótese à pessoa e como esse braço pode ser controlado. Para ele, os braços artificiais precisam ser o mais leves possível, ou então eles podem simplesmente ser deixados de lado pelo usuário.

\section{EXPERIMENTO}

Com o intuito de viabilizar o acesso a próteses biônicas de mão, este trabalho aponta resultados de pesquisas que envolvem a criação de próteses de baixo custo. Primeiramente, foi construída uma prótese de mão em uma impressora modelo Cubex, da 3D Systems (Figura 5), utilizando material polímero PLA. A movimentação dos dedos é realizada por meio de um par de cabos (um para flexão e outro para extensão) acionados por cinco servomotores de alto torque (12,5kg/ $\mathrm{cm})$ tipo MG946R da TowardPro. Esses servos recebem sinais de controle de um aplicativo móvel via Bluetooth. Cada um deles é responsável pelos movimentos de um dedo específico.

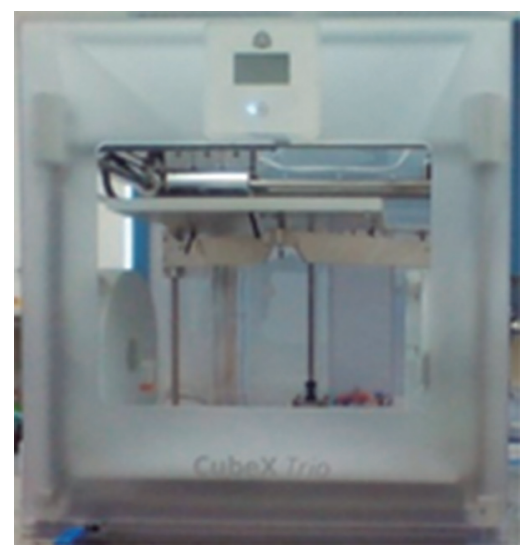

Figura 5. Impressora 3D Cubex.

Foi desenvolvido um aplicativo para o dispositivo móvel para gerenciar os sinais de controle enviados à prótese. Para a construção desse aplicativo foi necessário utilizar uma plataforma Software Development Kit (SDK), denominada Eclipse, específica para desenvolvimento de aplicativos Android. 
Lecheta (2013) descreve que o Android é uma nova plataforma de desenvolvimento para aplicativos móveis, baseada em um sistema operacional Linux, com diversas aplicações já instaladas, e, ainda, um ambiente de desenvolvimento bastante poderoso, ousado e flexível. A linguagem Java é utilizada na implementação do aplicativo em sistema Android, por possuir recursos disponíveis em multiplataformas, atendendo eficientemente a dispositivos móveis.

O componente de hardware usado para possibilitar a comunicação sem fios é um módulo Bluetooth HC-06 (Figura 6), conectado à placa Arduino para mediar a comunicação entre o dispositivo móvel e a prótese de mão.

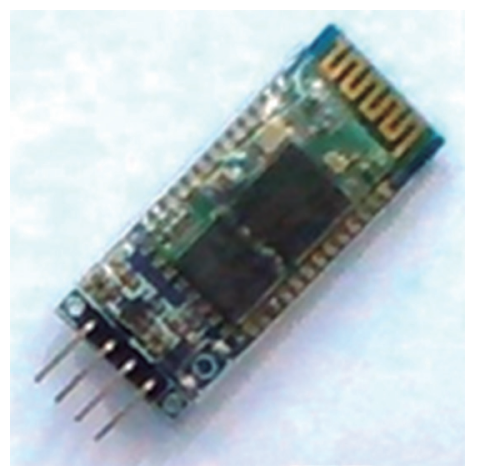

Figura 6. Módulo Bluetooth HC-06 utilizado.

A linguagem de programação Java possibilita o desenvolvimento de recursos acessíveis por módulo Bluetooth, utilizados para permitir o envio de sinais para a placa HC-06, por meio das seguintes bibliotecas: BluetoothAdapter, BluetoothDevice e BluetoothSocket.

Santos et al. (2012) explicam que o BluetoothAdapter representa, em linguagem de programação, o dispositivo físico no qual a aplicação está rodando, e o BluetoothDevice representa o dispositivo com o qual a aplicação deseja enviar sinais. Já o BluetoothSocket permite abrir uma conexão com dispositivos, possibilitando o início da comunicação para o envio de sinais.

Após a implementação em Java, foi acoplado ao Arduino um circuito eletrônico simplificado, composto por um diodo emissor de luz - Light Emitting Diode (LED) - e um resistor. Isso foi utilizado para testar a abertura da comunicação com a placa via Bluetooth por interface de dispositivo móvel. Na sequência, o próximo passo foi implementar um protótipo de tela em Java com dois botões que enviassem como sinal os caracteres 'A' ou 'B' (Figura 7).

Os botões implementados permitiam acender e apagar um LED. Como consequência da verificação de conexão de abertura da porta de comunicação, foi possível acoplar e controlar, simultaneamente, um servomotor teste de 5,5 V e adicionar uma barra de progresso para envio de rotação ao motor. 


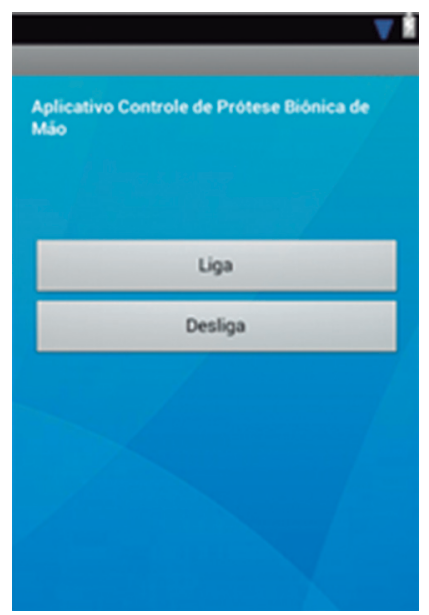

Figura 7. Interface do aplicativo de controle de prótese utilizado no experimento.

No caso do servomotor, o botão no aplicativo enviava os sinais de modo análogo ao caso dos primeiros botões, com a diferença de que, nesse caso específico, foram ajustados os ângulos de giro do servo por meio dos sinais enviados. Os passos de comunicação estão exibidos no fluxograma da Figura 8.

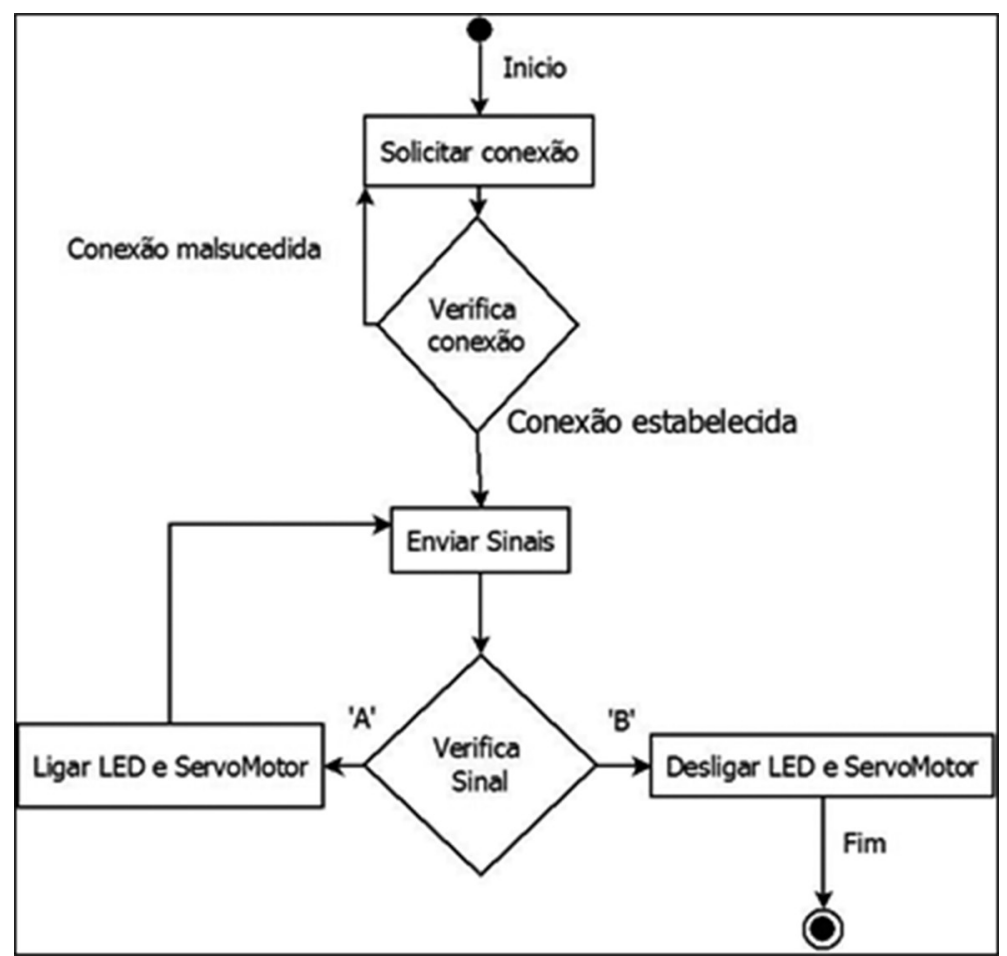

Figura 8. Passos da comunicação. 
A Figura 9 exibe a prótese utilizada e todo aparato de controle sem fio para os movimentos dos dedos, conforme descrito anteriormente.

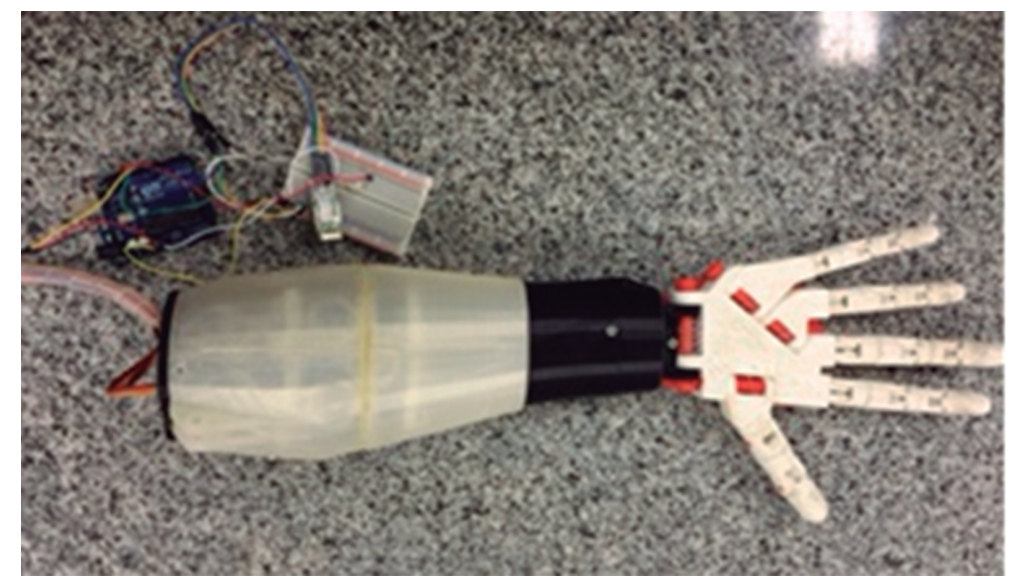

Figura 9. Prótese e demais componentes utilizados no experimento.

\section{RESULTADOS E CONCLUSÕES}

A conexão via Bluetooth, Arduino e dispositivo móvel apresentou resultado satisfatório no que diz respeito à comunicação entre interface física móvel e placa Arduino.

Com a utilização dos recursos BluetoothAdapter, BluetoothDevice e BluetoothSocket, foi possível capturar o endereço físico da placa Bluetooth HC-06, e assim utilizar esse endereço para a interface do aplicativo, o que permitiu o envio de sinais para a prótese.

Uma dificuldade encontrada durante a pesquisa foi a realização de carregamento e compilação do código do software processing para a placa Arduino. Ao usar os pinos 0 e 1 no Arduino para conexão dos terminais RX e TX da placa HC-06, o código não era compilado. Para resolver esse problema, foram definidas as portas 10 e 11 do Arduino para conexão com a placa Bluetooth. Assim foi possível liberar o carregamento do código.

Os testes realizados sugerem que o modelo desenvolvido apresenta características suficientes para a comunicação dos sistemas embarcados em dispositivos móveis para o controle de próteses Biônicas de mão. O próximo passo da pesquisa é implementar os mesmos recursos na linguagem Swift que permita ao usuário o controle de movimento por envio de sinais através de comandos de voz.

De acordo com a Apple (2015), o Swift é uma nova linguagem de programação intuitiva, desenvolvida pela empresa para a criação de aplicativos para dispositivos com iOS e MacOS. 
Novas implementações serão propostas com o objetivo de desenvolver barras de progresso na interface do aplicativo móvel para controle de dedos da prótese, possibilitando a implementação de uma aplicação para as plataformas Android e iOS, de modo a controlar de maneira eficaz os movimentos da prótese de mão.

\section{REFERÊNCIAS}

AMORIM, M.; GARRIDO, L. Mão biônica permite movimentos similares aos humanos. ADS. Disponível em: <http://professionals.ottobock.com.br/cps/rde/xbcr/ ob_br_pt/081204_Michelangelo.pdf> Acesso em: 16 jul. 2015.

APPLE. Swift. Uma linguagem aberta e poderosa, para todo mundo criar apps incríveis. Disponível em: <www.apple.com/br/swift>. Acesso em: 2 mai. 2015.

BANZI, M. Send in the clones. 2013. Disponível em: <https://blog.arduino. cc/2013/07/10/send-in-the-clones/>. Acesso em: 4 set. 2015.

BASTOS, B. L.; BORGES, M.; D’ABREU, J. Scratch, Arduino e o construcionismo: ferramentas para a educação. In: I STED - Seminário de Tecnologia Educacional de Araucária "Desafios e possibilidades para tecnologia educacional". Araucária, 2010. Anais. BRITO, V. A; OLIVEIRA, S. G.; CAETANO, J. L. Uma análise da implementação ZigBee pela tecnologia Sun SPOT. In: XXIX Congresso Da Sociedade Brasileira De Computação. Bento Gonçalves, 2009. Porto Alegre: Sociedade Brasileira de Computação, 2009. v. 1.

DELLON, B.; MATSUOKA, Y. Prosthetics, exoskeletons, and rehabilitation. IEEE Robotics \& Automation Magazine, v. 14, n. 1, p. 30-34, 2007.

EVANS, M.; NOBLE, J.; HOCHENBAUM, J. Arduino em ação. São Paulo: Novatec, 2013.

HUNOLD, M. et al. Protótipo de prótese de mão robótica de Lego controlada por sistema Android para bi-amputado. XXIV Congresso Brasileiro de Engenharia Biomédica - CBEB, 2014.

KUROSE, J. F.; ROSS, K. W. Redes de computadores e a internet: uma abordagem topdown. 5. ed. Tradução de Opportunity Translations, revisão técnica de Wagner Zucchi. São Paulo: Addison Wesley, 2010.

LECHETA, R. R. Google Android: aprenda a criar aplicações para dispositivos móveis com o Android SDK. 3. ed. São Paulo: Novatec, 2013.

LIMA, C. B.; VILLAÇA, M. A. M. Avr e Arduino. 2. ed. Florianópolis: Editora dos Autores, 2012.

LUCAS, J. What Is a Infrared. LiveScience. 2015. Disponível em: <http://www. livescience.com/50260-infrared-radiation.html>. Acesso em: 16 maio 2015.

MARGOLIS, M. Arduino Cook-book. 2. ed. Sebastopol: O’ Reilly Media, 2011.

McROBERTS, M. Arduino básico. Tradução de Rafael Zanolli. São Paulo: Novatec, 2011. 
MONK, S. Programação com Arduino: começando com Sketches. Tradução de Anatólio Laschuk. Porto Alegre: Bookman, 2013.

NORTON, K. M. A Brief History of Prosthetics. InMotion, vol. 17, nov./dez. 2007. Disponível em: <http://www.amputee-coalition.org/inmotion/nov_dec_07/history_ prosthetics.html>. Acesso em: 7 jul. 2015.

PRIESS, W. et al. Um mecanismo de escalonamento parametrizável para Scatternets Bluetooth. XXI Simpósio Brasileiro de Redes de Computadores, 2003.

SANTOS, D. et al. Controle de sistemas embarcados por sistema operacional móvel Android via Bluetooth. VII Coneepi - Congresso Norte e Nordeste de Pesquisa e Inovação. Palmas, 2012.

SARTORETTO, L.; BERSCH, R. O que é Tecnologia Assistiva. Assistiva Tecnologia e Educação, 2014. Disponível em <http://www.assistiva.com.br/tassistiva.html >. Acesso em: 15 maio 2015.

SCHIRMER, C. et al. Formação continuada a distância de professores para o atendimento educacional especializado. SEESP/SEED/MEC, 2007. Disponível em: <http://portal.mec.gov.br/seesp/arquivos/pdf/aee_df. pdf>. Acesso em: 30 abr. 2015. SIMÕES, L. Arduíno Original. Quando a pirataria começa? Quais os limites da OpenSource? Disponível em: <http://luizsimoes.com.br/pages/Artigos/Arduino_3.html>. Acesso em: 4 set. 2015.

TANENBAUM, A. S. Redes de computadores. Tradução de Vandenberg D. de Souza. 4. ed. Amsterdam: Campus, 2003.

TORRES, G. Redes de computadores. 2. ed. Rio de Janeiro: Novaterra, 2001.

WEBSTER, G. The bionic hand with the human touch. 2003. Disponível em: <http:// edition.cnn.com/2013/02/01/tech/bionichand-ilimb-prosthetic/>. Acesso em: 8 maio 2015.

WEIR, R. Design of artificial arms and hands for prosthetic applications. In: Standard Handbook of Biomedical Engineering and Design. Nova York: McGraw-Hill, 2004. 